Proc. Estonian Acad. Sci. Phys. Math., 2001, 50, 3, 133-144

\title{
FULLY DISCRETE COLLOCATION METHOD FOR WEAKLY SINGULAR INTEGRAL EQUATIONS
}

\author{
Enn TAMME
}

Institute of Applied Mathematics, University of Tartu, Liivi 2, 50409 Tartu, Estonia; enn.tamme@ut.ee

Received 5 April 2001

Abstract. To find the approximate solutions of a weakly singular integral equation by the collocation method it is necessary to solve linear systems whose coefficients are expressed as integrals. These integrals cannot usually be computed exactly. We get the fully discrete collocation method when we approximate the integrals by quadrature formulas on nonuniform grid. In this paper an appropriate grid is formed and the dependence of the convergence rate of this method on the choice of the grid is studied.

Key words: weakly singular integral equation, collocation method.

\section{INTRODUCTION}

Consider the integral equation

$$
u(x)=\int_{0}^{b} K(x, y) u(y) d y+f(x), \quad 0<x<b,
$$

whose kernel $K$ satisfies the following condition.

(A) The kernel $K(x, y)$ is $\gamma$ times $(\gamma \geq 1)$ continuously differentiable with respect to $x$ and $y$ for $x, y \in(0, b), x \neq y$, whereby there exists a real number $\nu \in(0,1)$ such that, for $x, y \in(0, b)$ and for all nonnegative integers $\alpha$ and $\beta$, with $\alpha+\beta \leq \gamma$, the inequality

$$
\left|\left(\frac{\partial}{\partial x}\right)^{\alpha}\left(\frac{\partial}{\partial x}+\frac{\partial}{\partial y}\right)^{\beta} K(x, y)\right| \leq c|x-y|^{-\nu-\alpha}
$$

holds. 
From the assumption (A) it follows that for $x, y \in(0, b), x \neq y$ and $\alpha+\beta \leq \gamma$ the inequality

$$
\left|\left(\frac{\partial}{\partial y}\right)^{\alpha}\left(\frac{\partial}{\partial x}+\frac{\partial}{\partial y}\right)^{\beta} K(x, y)\right| \leq c^{\prime}|x-y|^{-\nu-\alpha}
$$

holds too $\left[^{1}\right]$.

Such an integral equation can be effectively solved by the following piecewise polynomial collocation method on graded grids (see, e.g., $\left[{ }^{1}\right]$ ).

We divide the interval $[0, b]$ by the grid points

$$
\begin{aligned}
& x_{j}=\frac{b}{2}\left(\frac{j}{N}\right)^{r}, j=0,1, \ldots, N, \\
& x_{N+j}=b-x_{N-j}, j=1, \ldots, N,
\end{aligned}
$$

into $2 N \geq 2$ subintervals $\left[x_{j-1}, x_{j}\right], j=1, \ldots, 2 N$. Here the real number $r \geq 1$ characterizes the nonuniformity of the grid. If $r=1$, then the grid points are uniformly located. We choose $m$ points $\xi_{1}, \xi_{2}, \ldots, \xi_{m}$ in the interval $[-1,1]$ so that

$$
-1 \leq \xi_{1}<\xi_{2}<\ldots<\xi_{m} \leq 1
$$

and define in every subinterval $\left[x_{j-1}, x_{j}\right], j=1, \ldots, 2 N$, the collocation points

$$
\xi_{j q}=x_{j-1}+\frac{\xi_{q}+1}{2}\left(x_{j}-x_{j-1}\right), \quad q=1, \ldots, m .
$$

In every subinterval we search the approximate solution of Eq. (1) as the Lagrange interpolation polynomial of degree $m-1$

$$
u_{N}(x)=\sum_{q=1}^{m} u_{N}\left(\xi_{j q}\right) \varphi_{j q}(x), \quad x \in\left[x_{j-1}, x_{j}\right], \quad j=1, \ldots, 2 N,
$$

where

$$
\varphi_{j q}(x)=\prod_{\substack{p=1 \\ p \neq q}}^{m} \frac{x-\xi_{j p}}{\xi_{j q}-\xi_{j p}} .
$$

The values $u_{N}\left(\xi_{j p}\right)$ of the approximate solution we determine from the collocation condition

$$
u_{N}\left(\xi_{i p}\right)=\int_{0}^{b} K\left(\xi_{i p}, y\right) u_{N}(y) d y+f\left(\xi_{i p}\right), \quad i=1, \ldots, 2 N, \quad p=1, \ldots, m,
$$

or

$$
u_{N}\left(\xi_{i p}\right)=\sum_{j=1}^{2 N} \sum_{q=1}^{m} a_{i p j q} u_{N}\left(\xi_{j q}\right)+f\left(\xi_{i p}\right), \quad i=1, \ldots, 2 N, p=1, \ldots, m,
$$


where

$$
a_{i p j q}=\int_{x_{j-1}}^{x_{j}} K\left(\xi_{i p}, y\right) \varphi_{j q}(y) d y .
$$

Such a collocation method uses the values of the integrals (4) which usually cannot be computed analytically. A possibility of overcoming this difficulty is offered by the product integration method $\left[{ }^{2-4}\right]$. Using this method we need to compute exactly only the integrals containing the singular factor of the kernel $K(x, y)$. In this paper we replace the integrals (4) with quadrature sums discussed in $\left[^{5}\right]$. Such an approach is used also in $\left[^{6}\right]$.

\section{APPROXIMATION OF THE COEFFICIENTS (4)}

When computing the coefficients $a_{i p j q}$ it is necessary to take into account that in general the kernel $K\left(\xi_{i p}, y\right)$ is unbounded in the neighbourhood of $\xi_{i p}$. Therefore we use a nonuniform grid that is defined below. First we define the numbers

$$
\begin{gathered}
z_{k}=b_{1}\left(\frac{k}{M}\right)^{s}, \quad k=0,1, \ldots, M, \\
z_{M+k}=b_{1}\left(1+k \frac{s}{M}\right), \quad k=1,2, \ldots,
\end{gathered}
$$

where $0<b_{1} \leq b, M \geq N$, and $s>r$.

If $\xi_{i p} \in\left(x_{i-1}, x_{i}\right)$, then we present the coefficient $a_{i p i q}$ as the sum

$$
a_{i p i q}=a_{i p i q}^{-}+a_{i p i q}^{+},
$$

where

$$
a_{i p i q}^{-}=\int_{x_{i-1}}^{\xi_{i p}} K\left(\xi_{i p}, y\right) \varphi_{i q}(y) d y, \quad a_{i p i q}^{+}=\int_{\xi_{i p}}^{x_{i}} K\left(\xi_{i p}, y\right) \varphi_{i q}(y) d y .
$$

For the computation of the integrals in the intervals $\left(\xi_{i p}, x_{i}\right)$ and $\left(x_{j-1}, x_{j}\right)$, $j=i+1, \ldots, 2 N$, we define for fixed $i$ and $p$ the subintervals $\left(y_{k-1}, y_{k}\right)$ in the following way. We take

$$
y_{0}=\xi_{i p}, \quad y_{1}=y_{0}+\left(z_{1}-z_{0}\right), \quad y_{2}=y_{1}+\left(z_{2}-z_{1}\right),
$$

and so on until

$$
y_{k_{1}-1}=y_{k_{1}-2}+\left(z_{k_{1}-1}-z_{k_{1}-2}\right)<x_{i}
$$

and

$$
y_{k_{1}-1}+\left(z_{k_{1}}-z_{k_{1}-1}\right) \geq x_{i} .
$$


Then we take

$$
y_{k_{1}}=x_{i}, \quad y_{k_{1}+1}=y_{k_{1}}+\left(z_{k_{1}}-z_{k_{1}-1}\right),
$$

and so on until

$$
y_{k_{2}-1}=y_{k_{2}-2}+\left(z_{k_{2}-2}-z_{k_{2}-3}\right)<x_{i+1}
$$

and

$$
y_{k_{2}-1}+\left(z_{k_{2}-1}-z_{k_{2}-2}\right) \geq x_{i+1} .
$$

Then we take $y_{k_{2}}=x_{i+1}$. Continuing this way, we define the grid points $y_{k}$ as follows:

$$
y_{k_{j-i}}=x_{j-1}, \quad y_{k_{j-i}+1}=y_{k_{j-i}}+\left(z_{k_{j-i}-j+i+1}-z_{k_{j-i}-j+i}\right),
$$

and so on until

$$
y_{k_{j-i+1}-1}=y_{k_{j-i+1}-2}+\left(z_{k_{j-i+1}-j+i-1}-z_{k_{j-i+1}-j+i-2}\right)<x_{j}
$$

and

$$
y_{k_{j-i+1}-1}+\left(z_{k_{j-i+1}-j+i}-z_{k_{j-i+1}-j+i-1}\right) \geq x_{j} .
$$

Then we take $y_{k_{j-i+1}}=x_{j}$. We proceed in a similar way for $j=i+1, \ldots, 2 N$.

For the computation of the integrals (4) we choose a quadrature formula

$$
\int_{-1}^{1} \Phi(y) d y \approx \sum_{t=1}^{n} w_{t} \Phi\left(\eta_{t}\right)
$$

which is exact for all polynomials of degree $\mu, m-1 \leq \mu \leq 2 n-1$, and whose knots satisfy the conditions

$$
-1 \leq \eta_{1}<\eta_{2}<\ldots<\eta_{n} \leq 1 .
$$

The corresponding knots in the interval $\left[y_{k-1}, y_{k}\right]$ are

$$
\eta_{k t}=y_{k-1}+\frac{\eta_{t}+1}{2}\left(y_{k}-y_{k-1}\right), \quad t=1, \ldots, n .
$$

The approximate values $\tilde{a}_{i p i q}^{+}$and $\tilde{a}_{i p j q}$ of the integrals $a_{i p i q}^{+}$and $a_{i p j q}$ we compute by the formulas (cf. $\left[{ }^{5}\right]$ )

$$
\begin{aligned}
& \tilde{a}_{i p i q}^{+}=\sum_{k=2}^{k_{1}} \frac{y_{k}-y_{k-1}}{2} \sum_{t=1}^{n} w_{t} K\left(\xi_{i p}, \eta_{k t}\right) \varphi_{i q}\left(\eta_{k t}\right), \\
& \tilde{a}_{i p j q}=\sum_{k=k_{j-i}+1}^{k_{j-i+1}} \frac{y_{k}-y_{k-1}}{2} \sum_{t=1}^{n} w_{t} K\left(\xi_{i p}, \eta_{k t}\right) \varphi_{j q}\left(\eta_{k t}\right),
\end{aligned}
$$


where $j=i+1, \ldots, 2 N$ and $q=1, \ldots, m$. We use these formulas also in the case of $\xi_{i p}=x_{i-1}$. Then $\tilde{a}_{i p i q}=\tilde{a}_{i p i q}^{+}$. If $x_{i}-\xi_{i p}<z_{1}$, then we take $\tilde{a}_{i p i q}^{+}=0$ and by computation we replace with 0 the summand with the index $k=k_{1}+1=2$.

In an analogical way we compute the integrals also in the intervals $\left(x_{i-1}, \xi_{i p}\right)$ and $\left(x_{j-1}, x_{j}\right), j=i-1, i-2, \ldots, 1$. So we find the approximate values $\tilde{a}_{i p j q}$ for all coefficients $a_{i p j q}$ and form the fully discrete collocation method, in case of which we compute the approximate values $\tilde{u}_{N}\left(\xi_{i p}\right)$ of the solution of the integral equation (1) from the system of equations

$$
\begin{gathered}
\tilde{u}_{N}\left(\xi_{i p}\right)=\sum_{j=1}^{2 N} \sum_{q=1}^{m} \tilde{a}_{i p j q} \tilde{u}_{N}\left(\xi_{j q}\right)+f\left(\xi_{i p}\right), \\
i=1, \ldots, 2 N, \quad p=1, \ldots, m .
\end{gathered}
$$

We write the systems (3) and (7) in a more concise form:

$$
u_{N}=A_{N} u_{N}+f_{N}
$$

and

$$
\tilde{u}_{N}=\tilde{A}_{N} \tilde{u}_{N}+f_{N},
$$

where $A_{N}=\left(a_{i p j q}\right)$ and $\tilde{A}_{N}=\left(\tilde{a}_{i p j q}\right)$ are $2 \mathrm{Nm} \times 2 \mathrm{Nm}$ square matrices, and $u_{N}=\left(u_{N}\left(\xi_{i p}\right)\right), \tilde{u}_{N}=\left(\tilde{u}_{N}\left(\xi_{i p}\right)\right)$ and $f_{N}=\left(f\left(\xi_{i p}\right)\right)$ are vectors with $2 N m$ components. We use the norm of a vector

$$
\left\|u_{N}\right\|_{\infty}=\max _{\substack{1 \leq i \leq 2 N \\ 1 \leq p \leq m}}\left|u_{N}\left(\xi_{i p}\right)\right|
$$

and the corresponding norm of a matrix

$$
\left\|A_{N}\right\|_{\infty}=\max _{\substack{1 \leq i \leq 2 N \\ 1 \leq p \leq m}} \sum_{j=1}^{2 N} \sum_{q=1}^{m}\left|a_{i p j q}\right| .
$$

For the errors of the approximate values $\tilde{a}_{i p j q}$ of the coefficients $a_{i p j q}$ the following estimate holds.

Theorem 1. Let the kernel $K$ satisfy the assumption (A) with $\gamma=\mu-m+2$ and the quadrature formula (5) be exact for all polynomials of degree $\mu, m-1 \leq \mu<$ $2 n-1$, and $N \leq M$. Then

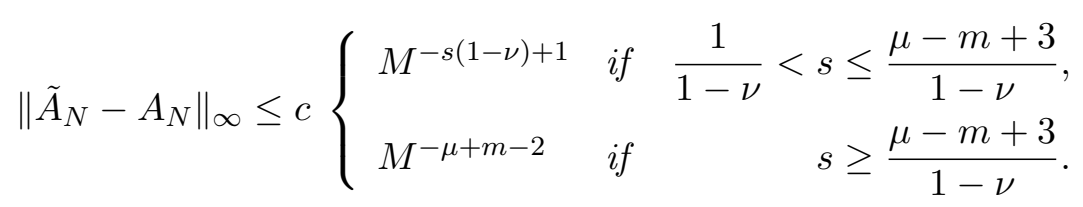


Proof. By change of the variable

$$
x=x_{j-1}+\frac{\xi+1}{2}\left(x_{j}-x_{j-1}\right)
$$

we get

$$
\begin{aligned}
\max _{\substack{1 \leq j \leq 2 N \\
1 \leq q \leq m}} \max _{x_{j-1} \leq x \leq x_{j}}\left|\varphi_{j q}(x)\right| & =\max _{\substack{1 \leq \leq \leq N \\
1 \leq q \leq m}} \max _{x_{j-1} \leq x \leq x_{j}}\left|\prod_{\substack{p=1 \\
p \neq q}}^{m} \frac{x-\xi_{j p}}{\xi_{j q}-\xi_{j p}}\right| \\
& =\max _{1 \leq q \leq m} \max _{-1 \leq \xi \leq 1}\left|\prod_{\substack{p=1 \\
p \neq q}}^{m} \frac{\xi-\xi_{p}}{\xi_{q}-\xi_{p}}\right|=\text { const. }
\end{aligned}
$$

We shall estimate the error of the approximate value $\tilde{a}_{i p j q}$ of $a_{i p j q}$ computed by (6). Let $k_{j-i}<k \leq k_{j-i+1}$, where $j-i \geq 0$ and $k_{0}=0$. Then $\left[y_{k-1}, y_{k}\right] \subset\left[x_{j-1}, x_{j}\right]$. As in $\left[^{5}\right]$ we can show that

$$
\begin{aligned}
\left|\mathcal{R}_{k}\right| & =\left|\int_{y_{k-1}}^{y_{k}} K\left(\xi_{i p}, y\right) \varphi_{j q}(y) d y-\frac{y_{k}-y_{k-1}}{2} \sum_{t=1}^{n} w_{t} K\left(\xi_{i p}, \eta_{k t}\right) \varphi_{j q}\left(\eta_{k t}\right)\right| \\
& \leq c \int_{y_{k-1}}^{y_{k}}\left|K\left(\xi_{i p}, y\right)-v(y)\right|\left|\varphi_{j q}(y)\right| d y
\end{aligned}
$$

where $v(y)$ is an arbitrary polynomial of degree $\mu-m+1 \geq 0$. With the letter $c$ we denote a constant whose value changes from time to time and which does not depend on $i, p, j, q, k, N$, and $M$. If $v$ is the Taylor polynomial

$$
v(y)=\sum_{\alpha=0}^{\mu-m+1} \frac{1}{\alpha !} \frac{\partial^{\alpha} K\left(\xi_{i p}, y_{k}\right)}{\partial y^{\alpha}}\left(y-y_{k}\right)^{\alpha},
$$

then for $k \geq 2$, due to (2), we estimate

$$
\begin{aligned}
\max _{y_{k-1} \leq y \leq y_{k}}\left|K\left(\xi_{i p}, y\right)-v(y)\right| & \leq \frac{\left|y_{k}-y_{k-1}\right|^{\mu-m+2}}{(\mu-m+2) ! y_{k-1} \leq y \leq y_{k}}\left|\frac{\partial^{\mu-m+2} K\left(\xi_{i p}, y\right)}{\partial y^{\mu-m+2}}\right| \\
& \leq c\left|y_{k-1}-\xi_{i p}\right|^{-\nu-\mu+m-2}\left|y_{k}-y_{k-1}\right|^{\mu-m+2}
\end{aligned}
$$

Thus, for $k \geq 2$

$$
\left|\mathcal{R}_{k}\right| \leq c\left|y_{k-1}-\xi_{i p}\right|^{-\nu-\mu+m-2}\left|y_{k}-y_{k-1}\right|^{\mu-m+3} .
$$


If $k-j+i \leq M$, then

$$
\begin{aligned}
& \left|y_{k}-y_{k-1}\right| \leq\left|z_{k-j+i}-z_{k-j+i-1}\right| \leq b_{1} s \frac{(k-j+i)^{s-1}}{M^{s}} \\
& \left|y_{k-1}-\xi_{i p}\right| \geq z_{k-j+i-1}=b_{1}\left(\frac{k-j+i-1}{M}\right)^{s} \geq 2^{-s}\left(\frac{k-j+i}{M}\right)^{s}
\end{aligned}
$$

and therefore

$$
\begin{aligned}
\left|\mathcal{R}_{k}\right| & \leq c M^{-s(1-\nu)}(k-j+i)^{s(1-\nu)-\mu+m-3} \\
& \leq c\left\{\begin{array}{lll}
M^{-s(1-\nu)} & \text { if } & s(1-\nu) \leq \mu-m+3, \\
M^{-\mu+m-3} & \text { if } & s(1-\nu) \geq \mu-m+3 .
\end{array}\right.
\end{aligned}
$$

But if $k-j+i>M$, then

$$
\begin{aligned}
& \left|y_{k}-y_{k-1}\right| \leq z_{k-j+i}-z_{k-j+i-1}=b_{1} \frac{s}{M}, \\
& \left|y_{k-1}-\xi_{i p}\right| \geq z_{M}=b_{1}
\end{aligned}
$$

and it follows from (10) that

$$
\left|\mathcal{R}_{k}\right| \leq c M^{-\mu+m-3} .
$$

As $M^{-\mu+m-3} \leq M^{-s(1-\nu)}$ if $s(1-\nu) \leq \mu-m+3$, we get for $i<j \leq 2 N$ and $x_{i}-\xi_{i p} \geq z_{1}$

$$
\left|\tilde{a}_{i p j q}-a_{i p j q}\right| \leq \sum_{k=k_{j-i}+1}^{k_{j-i+1}}\left|\mathcal{R}_{k}\right| \leq c\left(k_{j-i+1}-k_{j-i}\right) M^{-\min (s(1-\nu), \mu-m+3)} .
$$

Now we estimate

$$
\left|\tilde{a}_{i p i q}^{+}-a_{i p i q}^{+}\right| \leq\left|\int_{\xi_{i p}}^{y_{1}} K\left(\xi_{i p}, y\right) \varphi_{i q}(y) d y\right|+\sum_{k=2}^{k_{1}}\left|\mathcal{R}_{k}\right| .
$$

As

$$
\begin{aligned}
\left|\int_{\xi_{i p}}^{y_{1}} K\left(\xi_{i p}, y\right) \varphi_{i q}(y) d y\right| & \leq c \int_{y_{0}}^{y_{1}}\left(y-y_{0}\right)^{-\nu} d y \\
& =c \frac{\left(y_{1}-y_{0}\right)^{1-\nu}}{1-\nu} \leq c^{\prime} z_{1}^{1-\nu}=c^{\prime} b_{1}^{1-\nu} M^{-s(1-\nu)},
\end{aligned}
$$


taking into account the estimate for $\left|\mathcal{R}_{k}\right|$ we get

$$
\left|\tilde{a}_{i p i q}^{+}-a_{i p i q}^{+}\right| \leq c k_{1} M^{-\min (s(1-\nu), \mu-m+3)} .
$$

From these estimates it follows that

$$
\begin{aligned}
\sum_{q=1}^{m}\left|\tilde{a}_{i p i q}^{+}-a_{i p i q}^{+}\right|+ & \sum_{j=i+1}^{2 N} \sum_{q=1}^{m}\left|\tilde{a}_{i p j q}-a_{i p j q}\right| \\
& \leq c k_{2 N-i+1} M^{-\min (s(1-\nu), \mu-m+3)} .
\end{aligned}
$$

It is easy to check that these estimates hold also if $x_{i}-\xi_{i p}<z_{1}$.

Note that

$$
k_{2 N-i+1} \leq M+\left|b-b_{1}\right| \frac{M}{b_{1} s}+2 N+1 \leq c M .
$$

The errors of the approximations $\tilde{a}_{i p i q}^{-}$and $\tilde{a}_{i p j q}$ of $a_{i p i q}^{-}$and $a_{i p j q}, j=i-1$, $i-2, \ldots, 1$, are proved in an analogical way. This proves the theorem.

Remark. From the formulas (6) we see that the computation of the $4 m^{2} N^{2}$ elements of the matrix $\tilde{A}_{N}$ takes $O(N M)$ arithmetical operations.

\section{THE CONVERGENCE OF THE FULLY DISCRETE COLLOCATION METHOD}

We prove the following result.

Theorem 2. Assume that the quadrature formula (5) is exact for all polynomials of degree $\mu, m-1 \leq \mu \leq 2 n-1$ and the integral equation (1) has a unique solution $u \in L^{\infty}(0, b)$, whereas its kernel $K$ satisfies the assumption (A) with $\gamma=\max (m, \mu-m+2)$ and $f \in C^{m, \nu}(0, b)$ (see $\left.\left.{ }^{1,4}\right]\right)$. Then there exists $N_{0}>0$ so that for $M \geq N \geq N_{0}$ the system (7) has a unique solution $\tilde{u}_{N}=\left(\tilde{u}_{N}\left(\xi_{i p}\right)\right)$ and the following error estimate holds:

$$
\max _{\substack{1 \leq i \leq N \\ 1 \leq p \leq m}}\left|\tilde{u}_{N}\left(\xi_{i p}\right)-u\left(\xi_{i p}\right)\right| \leq c N^{-\min (m, s(1-\nu)-1)}
$$

if $r>\frac{m}{2(1-\nu)}$ and $\frac{1}{1-\nu}<s \leq \frac{\mu-m+3}{1-\nu}$.

If, in addition to these assumptions, the knots $\xi_{1}, \xi_{2}, \ldots, \xi_{m}$ satisfy the condition

$$
\int_{-1}^{1}\left(x-\xi_{1}\right)\left(x-\xi_{2}\right) \ldots\left(x-\xi_{m}\right) d x=0
$$


the kernel $K$ satisfies the assumption (A) with $\gamma=\max (m+1, \mu-m+2)$ and $f \in C^{m+1, \nu}(0, b)$, then, for $M \geq N \geq N_{0}$,

$$
\max _{\substack{1 \leq i \leq 2 N \\ 1 \leq p \leq m}}\left|\tilde{u}_{N}\left(\xi_{i p}\right)-u\left(\xi_{i p}\right)\right| \leq c N^{-\min (m+1-\nu, s(1-\nu)-1)}
$$

if $r>\frac{m}{1-\nu}$ and $\frac{1}{1-\nu}<s \leq \frac{\mu-m+3}{1-\nu}$.

Proof. It was proved in $\left[{ }^{1}\right]$ that under these assumptions there exists $N_{1}>0$ so that for $N \geq N_{1}$ there exists $\left(I-A_{N}\right)^{-1},\left\|\left(I-A_{N}\right)^{-1}\right\| \leq c$ and the collocation method (3) converges with the rate

$$
\left\|u_{N}-u\right\|_{\infty}=\max _{\substack{1 \leq i \leq 2 N \\ 1 \leq p \leq m}}\left|u_{N}\left(\xi_{i p}\right)-u\left(\xi_{i p}\right)\right| \leq c N^{-m} \quad \text { if } \quad r>\frac{m}{2(1-\nu)} .
$$

It follows from the equality

$$
I-\tilde{A}_{N}=\left(I-A_{N}\right)\left[I-\left(I-A_{N}\right)^{-1}\left(\tilde{A}_{N}-A_{N}\right)\right]
$$

and Theorem 1 that there exists $N_{0} \geq N_{1}$ so that for $N \geq N_{0}$ the system (9) has a unique solution $\tilde{u}_{N}=\left(\tilde{u}_{N}\left(\xi_{i p}\right)\right)$ and

$$
\left\|\tilde{u}_{N}\right\|_{\infty} \leq\left\|\left(I-\tilde{A}_{N}\right)^{-1}\right\|_{\infty}\left\|f_{N}\right\|_{\infty} \leq c .
$$

It follows from Eqs. (8) and (9) that

$$
\left(I-A_{N}\right)\left(\tilde{u}_{N}-u_{N}\right)=\left(\tilde{A}_{N}-A_{N}\right) \tilde{u}_{N} .
$$

Thus, for $N \geq N_{0}$,

$$
\begin{aligned}
\left\|\tilde{u}_{N}-u_{N}\right\|_{\infty} & \leq\left\|\left(I-A_{N}\right)^{-1}\right\|_{\infty}\left\|\tilde{A}_{N}-A_{N}\right\|_{\infty}\left\|\tilde{u}_{N}\right\|_{\infty} \\
& \leq c M^{-s(1-\nu)+1} \leq c N^{-s(1-\nu)+1}
\end{aligned}
$$

if $\frac{1}{1-\nu}<s \leq \frac{\mu-m+3}{1-\nu}$.

Using the inequality

$$
\left\|\tilde{u}_{N}-u\right\|_{\infty} \leq\left\|\tilde{u}_{N}-u_{N}\right\|_{\infty}+\left\|u_{N}-u\right\|_{\infty},
$$

we get the estimate (11).

Under the additional assumptions of Theorem 2 the collocation method (3) converges with the rate $\left[{ }^{1,4}\right]$

$$
\left\|u_{N}-u\right\|_{\infty} \leq c N^{-(m+1-\nu)} \quad \text { if } \quad r>\frac{m}{1-\nu} .
$$

From this the estimate (13) follows. 


\section{NUMERICAL EXAMPLE}

We consider the integral equation

$$
u(x)=\int_{0}^{1} \frac{u(y)}{\sqrt{|x-y|}} d y+f(x), \quad 0<x<1,
$$

where

$$
f(x)=\sqrt{x}-\sqrt{1-x}-\frac{\pi}{2} x-x \ln (1+\sqrt{1-x})+\frac{x}{2} \ln x .
$$

The unique solution of this equation is $u(x)=\sqrt{x}$. Here $f \in C^{\gamma, 0.5}(0,1)$ and the kernel $K(x, y)=|x-y|^{-1 / 2}$ satisfies the assumption (A) with $\nu=0.5$ for any $\gamma \geq 1$.

In Table 1 the norms of the errors

$$
\begin{aligned}
\left\|u_{N}-u\right\|_{\infty} & =\max _{\substack{1 \leq i \leq 2 N \\
1 \leq p \leq m}}\left|u_{N}\left(\xi_{i p}\right)-u\left(\xi_{i p}\right)\right|, \\
\left\|\tilde{u}_{N}-u\right\|_{\infty} & =\max _{\substack{1 \leq i \leq 2 N \\
1 \leq p \leq m}}\left|\tilde{u}_{N}\left(\xi_{i p}\right)-u\left(\xi_{i p}\right)\right|,
\end{aligned}
$$

and

$$
\left\|\tilde{A}_{N}-A_{N}\right\|_{\infty}=\max _{\substack{1 \leq i \leq 2 N \\ 1 \leq p \leq m}} \sum_{j=1}^{2 N} \sum_{q=1}^{m}\left|\tilde{a}_{i p j q}-a_{i p j q}\right|
$$

are presented. Here $u_{N}=\left(u_{N}\left(\xi_{i p}\right)\right)$ and $\tilde{u}_{N}=\left(\tilde{u}_{N}\left(\xi_{i p}\right)\right)$ are calculated by the (exact) collocation method (3) and by the fully discrete collocation method (7), respectively. Table 1 lists also the ratios of the errors calculated by dividing the norm corresponding to $N / 2$ by the norm corresponding to $N$. These ratios characterize the rate of convergence.

Table 1. The norms of the errors

\begin{tabular}{c|c|c|c|c|c|c}
\hline$N$ & $\left\|u_{N}-u\right\|_{\infty}$ & Ratio & $\left\|\tilde{u}_{N}-u\right\|_{\infty}$ & Ratio & $\left\|\tilde{A}_{N}-A_{N}\right\|_{\infty}$ & Ratio \\
\hline 4 & $2.2 \mathrm{E}-2$ & & $2.4 \mathrm{E}-2$ & & $5.5 \mathrm{E}-3$ & \\
8 & $3.6 \mathrm{E}-3$ & 6.0 & $3.6 \mathrm{E}-3$ & 6.6 & $4.8 \mathrm{E}-4$ & 11.6 \\
16 & $3.4 \mathrm{E}-4$ & 10.6 & $3.4 \mathrm{E}-4$ & 10.7 & $4.3 \mathrm{E}-5$ & 11.1 \\
32 & $2.5 \mathrm{E}-5$ & 13.3 & $2.5 \mathrm{E}-5$ & 13.6 & $3.8 \mathrm{E}-6$ & 11.4 \\
64 & $1.8 \mathrm{E}-6$ & 14.4 & $1.8 \mathrm{E}-6$ & 14.3 & $3.3 \mathrm{E}-7$ & 11.4 \\
128 & $1.2 \mathrm{E}-7$ & 15.0 & $1.3 \mathrm{E}-7$ & 13.8 & $3.0 \mathrm{E}-8$ & 11.1
\end{tabular}


For calculating $u_{N}$ and $\tilde{u}_{N}, r=4$ is chosen and the knots of Simpson's rule are used as the collocation points. Then $m=3, \xi_{1}=-1, \xi_{2}=0, \xi_{3}=1$, and the systems (3) and (7) contain $4 N+1$ equations and unknowns. Since these knots satisfy the condition (12), the estimate (14) guarantees the convergence of the (exact) collocation method with the rate $O\left(N^{-3.5}\right)$ for $r>6$ only. The ratio ought to be approximately $2^{3.5} \approx 11.3$. From Table 1 we see that already in the case of $r=4$ the collocation method converges faster. If $r=5$, the errors are greater, and if $r=6$, they are still greater for all values of $N$, though the rate of convergence is nearly the same.

For computation of $\tilde{A}_{N}$ and $\tilde{u}_{N}$ we choose $s=7, b_{1}=0.5, M=4 N$, and the Gaussian quadrature with 3 knots for numerical integration. As seen from Table 1, the approximate solutions obtained by the fully discrete collocation method and by the collocation method have nearly the same accuracy. The convergence, however, is somewhat quicker than Theorems 1 and 2 guaranteed.

Note that in this example we can calculate the coefficients $a_{i p j q}$ as differences of the corresponding antiderivatives, but then a significant loss of accuracy due to round-off errors occurs for $N=64$ and especially for $N=128$. For getting the results presented in Table 1 we reduced the round-off errors by transformation of these differences. In the calculation of the coefficients $\tilde{a}_{i p j q}$ by formulas (6) the round-off errors were smaller and it was not necessary to modify the formulas.

\section{ACKNOWLEDGEMENT}

This investigation was supported by the Estonian Science Foundation under grant No. 4410.

\section{REFERENCES}

1. Vainikko, G. Multidimensional weakly singular integral equations. Lecture Notes in Math., 1993, 1549.

2. Schneider, C. Product integration for weakly singular integral equations. Math. Comp., 1981, 36, 207-213.

3. Atkinson, K. E. The Numerical Solution of Integral Equations of the Second Kind. Cambridge Univ. Pr., 1997.

4. Tamme, E. The discrete collocation method for weakly singular integral equations. In Differential and Integral Equations: Theory and Numerical Analysis (Pedas, A., ed.). Estonian Math. Soc., Tartu, 1999, 97-105.

5. Tamme, E. Numerical computation of weakly singular integrals. Proc. Estonian Acad. Sci. Phys. Math., 2000, 49, 215-224.

6. Kaneko, H. and $\mathrm{Xu}, \mathrm{Y}$. Gauss-type quadratures for weakly singular integrals and their application to Fredholm integral equations of second kind. Math. Comp., 1994, 62, 739-753. 


\section{TÄIELIKULT DISKREETNE KOLLOKATSIOONIMEETOD NÕRGALT SINGULAARSETE INTEGRAALVÕRRANDITE LAHENDAMISEKS}

\section{Enn TAMME}

Nõrgalt singulaarse integraalvõrrandi lähislahendi leidmisel kollokatsioonimeetodiga tuleb lahendada lineaarseid võrrandisüsteeme, mille kordajad avalduvad integraalidena. Neid integraale saab vaid erandjuhtudel täpselt leida. Täielikult diskreetse kollokatsioonimeetodini jõuame, kui lähendame neid integraale kvadratuurvalemite abil ebaühtlasel võrgul. Artiklis on moodustatud sobiv võrk ja selgitatud meetodi koonduvuskiiruse sõltuvus võrgu valikust. 